\title{
The "growing teratoma syndrome" in primary mediastinal nonseminomatous germ cell tumors: Criteria based on current practice
}

\author{
Kenneth A. Kesler, MD, ${ }^{a}$ Jay B. Patel, MD, ${ }^{a}$ Laura E. Kruter, MD, ${ }^{\text {a }}$ Thomas J. Birdas, MD, ${ }^{\text {a }}$ \\ Karen M. Rieger, MD, ${ }^{a}$ Ikenna C. Okereke, MD, ${ }^{a}$ and Lawrence H. Einhorn, MD $^{\mathrm{b}}$
}

\begin{abstract}
Objective: Criteria for the growing teratoma syndrome in patients with primary mediastinal nonseminomatous germ cell tumors have not been well established according to current practice.

Methods: An institutional database identified 188 patients who underwent postchemotherapy surgery for primary mediastinal nonseminomatous germ cell tumors from 1981 to 2009. We reviewed the subset of patients who underwent urgent surgery for tumor growth resulting in cardiopulmonary deterioration secondary to mediastinal compression precluding safe completion of 4 cisplatin-based chemotherapy cycles with rapidly declining serum tumor markers.
\end{abstract}

\begin{abstract}
Results: Five men (2.6\%) with an average age of 25.8 years were identified. All patients initially presented with a large symptomatic anterior mediastinal mass and elevated serum tumor markers. Patients received an average of 2.4 chemotherapy cycles of a scheduled 4 courses before cardiopulmonary deterioration. Pathology of the resected specimens demonstrated mature teratoma in all patients; however, it was admixed in 4 patients with foci of immaturity $(n=1)$, malignant transformation of teratoma to sarcoma $(n=2)$, and nonseminomatous germ cell tumor $(n=2)$. There was 1 operative death. Three of the 4 operative survivors subsequently completed a total of 4 cycles of chemotherapy after recovery. Two patients are alive and well after an average of 14 years. Two patients died of metastatic disease.
\end{abstract}

Conclusions: The growing teratoma syndrome should be defined not only as a growing mediastinal mass but also with secondary cardiopulmonary deterioration precluding safe completion of planned chemotherapy in the presence of declining serum tumor markers. Prompt recognition of this syndrome, discontinuation of chemotherapy, and surgical intervention can result in cure. (J Thorac Cardiovasc Surg 2012;144:438-43)

Nonseminomatous germ cell tumors frequently demonstrate mixed histology. Common to all nonseminomatous germ cell tumors are malignant elements that are sensitive to cisplatin-based chemotherapy, including yolk sac carcinoma, embryonal carcinoma, or choriocarcinoma, in order of frequency. An admixture of varying proportions usually is also present with elements that are not sensitive to chemotherapy, including mature teratoma, immature teratoma, or, on occasion, frank malignant degeneration of teratoma into nongerm cell cancers, such as sarcoma. The term "growing teratoma syndrome" (GTS) was first coined by Logothetis and colleagues, ${ }^{1}$ reporting on patients with nonseminomatous testicular cancer and growing retroperitoneal or lung masses

From the Cardiothoracic Division, ${ }^{\mathrm{a}}$ Department of Surgery, Melvin and Bren Simon Cancer Center, Indiana University Health, Indianapolis, Ind; and Hematology/Oncology Division, ${ }^{\mathrm{b}}$ Department of Medicine, Melvin and Bren Simon Cancer Center, Indiana University Health, Indianapolis, Ind.

Disclosures: Authors have nothing to disclose with regard to commercial support.

Received for publication Feb 2, 2012; revisions received March 31, 2012; accepted for publication May 16, 2012; available ahead of print June 19, 2012.

Address for reprints: Kenneth A. Kesler, MD, Indiana University Department of Surgery, Cardiothoracic Division, Barnhill Drive EM \#212, Indianapolis, IN 46202 (E-mail: kkesler@iupui.edu).

0022-5223/\$36.00

Copyright (c) 2012 by The American Association for Thoracic Surgery doi:10.1016/j.jtcvs.2012.05.053 during observation after chemotherapy, and included the following criteria: normalization of previously elevated serum tumor markers, "paradoxical" increase in tumor size, and absence of any pathologic component other than mature teratoma in the surgical specimen. ${ }^{1}$ Since this definition in 1982, the treatment paradigm for nonseminomatous germ cell tumors with cisplatin-based chemotherapy followed by surgical resection of residual disease as a planned approach in select patients has evolved and is considered one of the most successful models of multimodality cancer therapy.

It has been well established that primary mediastinal nonseminomatous germ cell tumors (PMNSGCT), although histologically identical to their more common testicular counterparts, have a distinctly inferior overall prognosis and therefore have been designated as "poor risk" nonseminomatous germ cell tumors along with other subsets of testicular nonseminomatous germ cell tumors. ${ }^{2}$ Although metastatic testicular nonseminomatous germ cell tumors have an overall 5-year survival exceeding $80 \%$ with cisplatin-based chemotherapy, their mediastinal counterparts demonstrate a more aggressive biology with 5-year survivals averaging between $40 \%$ and $50 \%{ }^{3,4}$ Factors that contribute to inferior survival include an overall poorer response to chemotherapy and higher incidence of degenerative nongerm cell cancer pathology in the 


\section{Abbreviations and Acronyms \\ AFP = alpha-fetoprotein \\ BEP = bleomycin, etoposide, cisplatin \\ CT = computed tomography \\ GTS = growing teratoma syndrome \\ PMNSGCT $=$ primary mediastinal \\ nonseminomatous germ cell tumor}

residual mass. Because of these factors and the poor response to second-line chemotherapy, surgical removal of any residual disease after recovery from cisplatin-based chemotherapy as a planned approach for PMNSGCT has been advocated. $^{5-7}$ In this study, we review our institutional experience with the subset of patients with PMNSGCT who demonstrated tumor growth during chemotherapy despite a decrease in serum tumor marker levels (alphafetoprotein [AFP] and human chorionic gonadotropin), which resulted in cardiopulmonary deterioration secondary to mediastinal compression precluding safe completion of 4 cisplatin-based chemotherapy cycles, mandating urgent surgery.

\section{MATERIAL AND METHODS}

After obtaining formal institutional approval, a prospective database search initially identified 188 patients who underwent surgery at the Melvin and Bren Simon Cancer Center for PMNSGCT after cisplatin-based chemotherapy from 1981 to 2009 . Of these, a subset of 5 patients $(2.6 \%)$ demonstrated tumor growth during chemotherapy with a rapid decrease in serum tumor marker levels. This resulted in cardiopulmonary deterioration secondary to mediastinal compression precluding safe completion of or recovery from 4 cisplatin-based chemotherapy cycles, mandating urgent surgery. These 5 patients constitute the basis of this study. Institutional records or records provided by referring facilities were reviewed. Multiple demographic, pathologic, operative, and outcome variables were recorded. ${ }^{8}$

\section{RESULTS}

All 5 patients were healthy men aged 20 to 33 years (mean, 25.8 years). All patients presented at the time of diagnosis with a large symptomatic anterior mediastinal mass and elevated AFP at an average of $9137 \mathrm{ng} / \mathrm{mL}$ (range, 791$36,000 \mathrm{ng} / \mathrm{mL}$ ), diagnostic of a PMNSGCT. Human chorionic gonadotropin was elevated in 3 patients with a mean of $206 \mathrm{mIU} / \mathrm{mL}$ (range, $8-350 \mathrm{mIU} / \mathrm{mL}$ ). Computed tomography (CT) evidence of metastatic disease (lung) was present in 1 patient at the time of diagnosis, with the remaining patients presenting with disease isolated to the mediastinum. The specifics of all cases are described next and in Table 1. Prechemotherapy biopsies demonstrated mature teratoma in only 2 patients despite elevated AFP, mature teratoma, and foci of nonseminomatous germ cell tumor in 2 patients and 1 patient with immature teratoma and foci of nonseminomatous germ cell tumor. Patients received an average of 2.4 cycles (range, 1-3) of cisplatin-based chemotherapy before undergoing surgery. Three patients had normalized serum tumor markers, and 2 patients demonstrated rapid serum tumor marker decline before surgery.

The details of our surgical approach have been described. ${ }^{7,9}$ In brief, the effectiveness of cisplatin-based chemotherapy for germ cell cancer usually results in extensive tumor necrosis that is more marked around the periphery. This finding usually allows a complete resection that minimizes operative morbidity by preserving critical structures that abut but are not densely adherent to or directly involved with the residual mass, such as lung, great veins, phrenic nerves, and occasionally cardiac chambers where the "pericardial barrier" has been violated. In general, we believe an aggressive but balanced dissection to remove all residual disease while sparing critical structures with generous use of frozen-section analysis is warranted. Choosing the surgical approach that provides optimal exposure of residual disease and surrounding structures is initially critical. A bilateral anterior thoracotomy with transverse sternotomy (also called the "clamshell" incision) is used for large midline tumors with significant extension into the chest. Finally, cardiopulmonary bypass capabilities are available for cases with large or invasive-appearing tumors, such as in the patients reported in this series. Four patients underwent a surgery through a clamshell approach. One patient required an initial median sternotomy and then a sequential left lateral thoracotomy. All 5 patients underwent complete surgical extirpation of the mediastinal mass with tumor-free margins. The 2 patients who had not normalized but demonstrated rapid serum tumor marker decline at the time of surgery had normalized tumor markers by the time of hospital discharge.

Surgical pathology of the mediastinal mass demonstrated mature teratoma only and mature teratoma with focal immaturity in 1 patient each. Pathology was mixed in the other 3 patients with predominately mature teratoma and focal yolk sac cancer $(n=1)$, nongerm cell cancer (angiosarcoma, $\mathrm{n}=1$ ), and a combination of yolk sac and nongerm cell cancer (angiosarcoma, $\mathrm{n}=1$ ). One patient died of respiratory failure postoperatively. Three of the 4 surviving patients received cisplatin-based chemotherapy after recovery to complete 4 cycles. All 4 surviving patients recovered good to excellent performance status. Two patients are alive and well 13 and 15 years after initial surgery; however, both have required further metastasectomies during follow-up. The other 2 patients died of metastatic nongerm cell cancer after 20 and 26 months.

\section{Patient 1}

A 22-year-old man presented with dyspnea and left-sided chest discomfort. CT demonstrated a large anterior mediastinal mass ( $23 \mathrm{~cm}$ in longest dimension) compressing the trachea, heart, and great vessels with almost total obliteration of 
TABLE 1. Prechemotherapy and postchemotherapy pathology, serum tumor markers at diagnosis, and status at last follow-up

\begin{tabular}{|c|c|c|c|c|c|c|c|}
\hline Patient no. & Pathology at diagnosis & $\begin{array}{c}\text { AFP } \\
(\mathbf{n g} / \mathbf{m L})\end{array}$ & $\begin{array}{c}\text { hCG } \\
(\mathbf{m I U} / \mathbf{m L})\end{array}$ & $\begin{array}{c}\text { Cycles of } \\
\text { chemotherapy }\end{array}$ & Surgical pathology & Status & $\begin{array}{l}\text { Length of } \\
\text { follow-up }\end{array}$ \\
\hline 1 & $\begin{array}{l}\text { Immature teratoma with } \\
\text { focal yolk sac and } \\
\text { embryonal carcinoma }\end{array}$ & 5131 & Normal & 2 & $\begin{array}{l}\text { Mature teratoma with } \\
\text { focal immature teratoma }\end{array}$ & $\begin{array}{l}\text { Alive and well } \\
\text { (3 metastasectomies) }\end{array}$ & $15 \mathrm{y}$ \\
\hline 2 & Mature teratoma & 791 & 350 & 3 & $\begin{array}{l}\text { Mature teratoma with focal } \\
\text { yolk sac cancer }\end{array}$ & $\begin{array}{l}\text { Alive and well } \\
\text { (1 metastasectomy) }\end{array}$ & $13 \mathrm{y}$ \\
\hline 3 & $\begin{array}{l}\text { Mature teratoma with } \\
\text { focal yolk sac } \\
\text { carcinoma }\end{array}$ & 961 & Normal & 3 & $\begin{array}{l}\text { Mature teratoma with focal } \\
\text { angiosarcoma and yolk sac } \\
\text { carcinoma }\end{array}$ & Died of disease & $1.7 \mathrm{y}$ \\
\hline 4 & $\begin{array}{l}\text { Mature teratoma with } \\
\text { focal yolk sac } \\
\text { carcinoma }\end{array}$ & 2800 & 8 & 3 & $\begin{array}{l}\text { Mature teratoma with focal } \\
\text { angiosarcoma }\end{array}$ & Operative death & $36 \mathrm{~d}$ \\
\hline 5 & Mature teratoma & 36,000 & 261 & 1 & Mature teratoma & Died of disease & $2.2 \mathrm{y}$ \\
\hline
\end{tabular}

$A F P$, Alpha-fetoprotein; $h C G$, human chorionic gonadotropin.

the left pleural space. An open biopsy at an outside hospital was consistent with predominately immature teratoma and a small focus of embryonal cell carcinoma. He received 2 cycles of bleomycin, etoposide, cisplatin (BEP) chemotherapy, but symptoms of further respiratory distress and upper-body edema consistent with superior vena cava obstruction developed. Repeat CT revealed an increase in the size of the lesion with further cardiopulmonary compression (Figure 1). Serum AFP was rapidly declining. He was then referred to the Melvin and Bren Simon Cancer Center for urgent surgery, which was performed through a clamshell approach. The mass was excised requiring en bloc pericardiectomy, a small area of diaphragm, and wedge resection of the left upper lobe and left phrenic nerve. Final pathology revealed

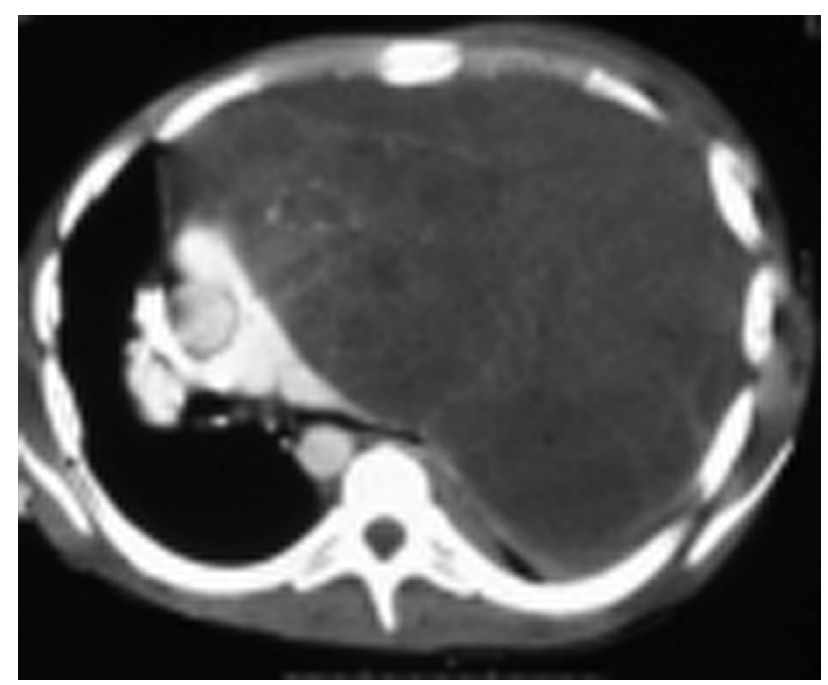

FIGURE 1. Preoperative axial CT of patient 1 demonstrating a large heterogeneous anterior mediastinal mass with cardiac/great vessel displacement and compression. The left pleural space is also obliterated. A leftward clamshell approach provided adequate exposure to the anterior mediastinum and pleural space for surgical removal. mature teratoma with foci of immaturity. He received 2 additional cycles of cisplatin-based chemotherapy after recovery. Serum tumor marker levels remained normal; however, 2 new left-sided pulmonary nodules were identified on CT scan after 3 years of follow-up. Both nodules were excised and pathologically demonstrated mature teratoma only. Three years after the second operation, posterior flank pain developed in the patient, with CT revealing a mass involving the chest wall in the left posterior costophrenic sulcus associated with slight elevation of AFP. Fine-needle biopsy demonstrated teratoma with foci of high-grade leiomyosarcoma for which the patient underwent wide chest wall resection and reconstruction. Seven years later, 13 years after the initial operation, another symptomatic recurrence with a high-grade sarcoma involving a lower thoracic vertebral body developed in the patient, for which he underwent vertebrectomy. The findings of sarcoma in the chest wall and spine represented malignant transformation of teratoma. At 15 years after the initial operation, the patient shows no evidence of disease.

\section{Patient 2}

A 30-year-old man presented with a cough, left-sided chest discomfort, and progressive shortness of breath. CT demonstrated a large anterior mediastinal mass $(15 \mathrm{~cm}$ in longest direction) obliterating two thirds of the left pleural space with compression of the heart and great vessels along with 2 pulmonary nodules suspicious for metastatic disease. Fine-needle aspiration biopsy at an outside hospital demonstrated only mature teratoma; however, significant elevation of serum tumor markers indicated a malignant nonseminomatous component. After completion of the third cycle of BEP chemotherapy, repeat imaging demonstrated an increase in the mediastinal mass and serum tumor markers had normalized. His shortness of breath was occurring with minimal activity, and he was referred to the Melvin and Bren Simon Cancer Center for surgical therapy before 
receiving the fourth chemotherapy cycle. He was taken to surgery, where excision requiring en bloc pericardiectomy via a clamshell approach was performed. No pulmonary resection was required to remove the mediastinal mass; however, both lung nodules were removed separately. Final pathology of the mediastinal mass revealed mature teratoma with a focus of yolk sac carcinoma. The pulmonary nodules pathologically demonstrated only mature teratoma. He received 2 additional cycles of cisplatin-based chemotherapy after recovery. One year after initial surgery, a symptomatic recurrence involving a lower thoracic vertebra developed, and he underwent vertebrectomy with pathology revealing mature teratoma. At 13 years after the initial operation, the patient shows no evidence of disease.

\section{Patient 3}

A 33-year-old man presented with symptoms of right shoulder pain, dyspnea, and dysphagia. CT demonstrated a large (15 cm in longest direction) anterior mediastinal mass with compression of the heart, great vessels, and trachea. Fine-needle aspiration biopsy at an outside hospital confirmed the diagnosis of mature teratoma with foci of yolk sac carcinoma. AFP was significantly elevated. After completing the third round of BEP chemotherapy, the patient experienced acute respiratory decompensation, stridor, and symptoms of superior vena cava obstruction. Repeat CT revealed a notable increase in the size of the mass with almost complete tracheal occlusion from extrinsic compression. Serum tumor markers had normalized. He was referred to the Melvin and Bren Simon Cancer Center for urgent surgery. The mass was excised including en bloc pericardiectomy, resection of the right phrenic nerve and left innominate vein, and right upper and middle lobectomies through a clamshell approach. Final pathology revealed mature teratoma with foci of angiosarcoma and yolk sac carcinoma. Widespread angiosarcoma metastases developed in the patient, and he died 20 months after surgery.

\section{Patient 4}

A 23-year-old presented with symptoms of dyspnea, right-sided chest pain, and cough. CT demonstrated a large (15 cm in longest direction) anterior mediastinal mass with compression of the heart, great vessels, and almost total obliteration of the right pleural space. Open biopsy at an outside hospital showed mature teratoma with foci of yolk sac carcinoma. After completing the third round of BEP chemotherapy, the patient's shortness of breath significantly worsened with repeat CT demonstrating an increase in tumor dimensions and further mediastinal compression. Serum tumor markers were normalizing appropriately. He was referred to the Melvin and Bren Simon Cancer Center for urgent surgery, which required en bloc extrapleural right pneumonectomy for extensive lung and hilar involvement, and resection of the right phrenic nerve and pericardium through a clamshell approach. Final pathology revealed mainly mature teratoma with foci of angiosarcoma. On the fourth postoperative day, the patient manifested respiratory failure secondary to "postpneumonectomy pulmonary edema," and he died on postoperative day 36.

\section{Patient 5}

A 20-year-old presented with left shoulder pain and dyspnea. CT demonstrated a large ( $26 \mathrm{~cm}$ in longest dimension) anterior mediastinal mass compressing the heart, great vessels, and complete obliteration of the left pleural space with inferior displacement of the diaphragm. Fine-needle biopsy demonstrated only mature teratoma; however, serum tumor markers were significantly elevated, diagnostic of PMNSGCT. After completing the first BEP cycle, respiratory decompensation developed in the patient. He was transferred to the Melvin and Bren Simon Cancer Center with $\mathrm{CT}$ showing an increase in tumor size and rapidly declining serum tumor markers (Figure 2). The patient required intubation shortly after transfer and underwent emergency surgical resection of the mediastinal mass requiring en bloc left upper lobectomy, resection of the left phrenic nerve, and subtotal pericardiectomy. It was thought that a clamshell approach would not provide adequate inferior exposure; therefore, initial dissection was performed through a median sternotomy followed by a separate left posterolateral thoracotomy to complete the resection. Final pathology again revealed only mature teratoma. The patient received the remaining 3 cycles of cisplatin-based chemotherapy after

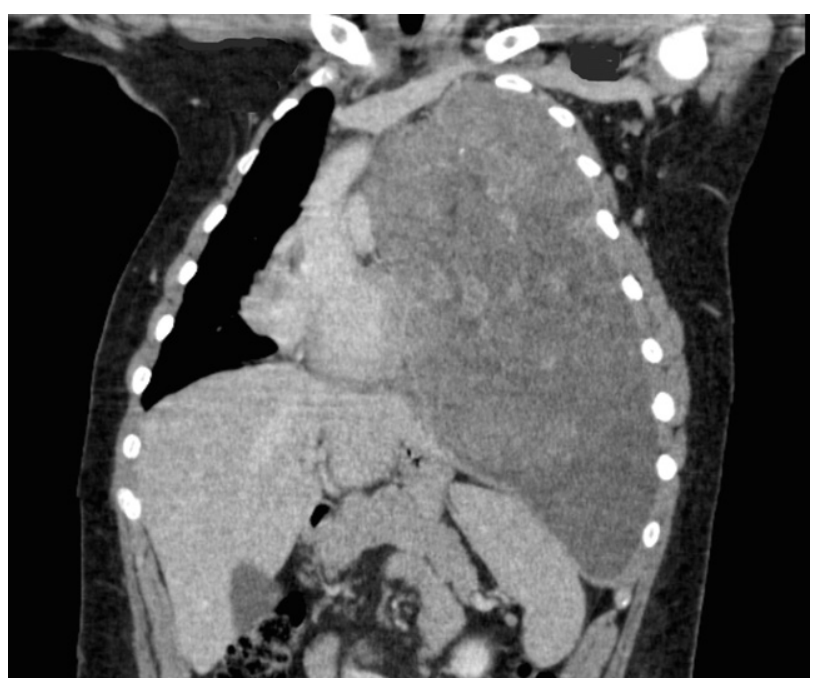

FIGURE 2. Preoperative coronal CT of patient 5 demonstrating a large heterogeneous anterior mediastinal mass with cardiac/great vessel displacement and compression. The left pleural space is also obliterated. Because of significant inferior extension, surgical removal required an initial sternotomy and sequential left thoracotomy. 
recovery, but he died of metastatic immature teratoma/ sarcoma 26 months after surgery.

\section{DISCUSSION}

Given the "poor risk" designation of PMNSGCT, we have maintained a policy of surgically removing any residual disease as a planned approach 4 to 6 weeks after completing 4 cycles of cisplatin-based chemotherapy, which allows adequate functional and hematologic recovery before a major thoracic surgical procedure. Pathologic analysis of 158 patients with PMNSGCT undergoing surgery after chemotherapy at the Melvin and Bren Simon Cancer Center revealed complete tumor necrosis in $25 \%$ and teratoma in $33 \%$. ${ }^{9}$ The remaining $41 \%$ of patients pathologically demonstrated elements of malignancy in the form of persistent germ cell or non-germ cell cancer, with the majority of these patients also having mixed pathology, including teratoma in the surgical specimen. Theoretically therefore, if patients with PMNSGCT were observed after completing chemotherapy, only a small fraction would demonstrate a stable residual mediastinal mass, with the majority developing interval growth with normalized serum tumor markers (mature teratoma, immature teratoma, or nongerm cell cancer) or interval growth with increasing serum tumor markers (nonseminomatous germ cell cancer). Unfortunately, there is no role for postchemotherapy positron emission tomography scanning to determine the need for removal of a residual mass, because teratoma does not demonstrate hypermetabolic activity. Positron emission tomography also lacks the sensitivity to identify microscopic foci of persistent nonseminomatous germ cell or nongerm cell cancer.

The majority of PMNSGCTs occur in young adult men. Most patients present symptomatically with chest pain, cough, superior vena cava syndrome, or shortness of breath secondary to a rapidly growing anterior mediastinal mass. CT usually demonstrates a large heterogeneous mass with occasional evidence of necrosis and hemorrhage. Associated pericardial and pleural effusions are also common, contributing to the symptoms, but typically not malignant in nature. Because of the sensitivity of nonseminomatous germ cell cancer to cisplatin-based chemotherapy, there is normally a significant decrease in the size of the mediastinal neoplasm, decrease in serum tumor marker levels, and improvement in the patient's respiratory status after chemotherapy is initiated. Because PMNSGCTs typically demonstrate mixed histology, the response to chemotherapy is proportional to the amount of chemosensitive pathology within the individual neoplasm. For unusual patients with significant amounts of pathology contained in the primary neoplasm, which are not sensitive to chemotherapy, "paradoxical" growth with decreasing serum tumor markers may occur during chemotherapy. Pathology in PMNSGCT that is not sensitive to chemotherapy and typically does not produce serum tumor markers would include not only mature teratoma but also immature teratoma and degenerative non-germ cell cancer. Although biopsy is not necessary to establish a diagnosis of PMNSGCT, the finding that all patients in our GTS series pathologically demonstrated mature teratoma or predominately mature teratoma with foci of other mixed histology in prechemotherapy biopsy supports this concept. In patients with large symptomatic neoplasms at the time of presentation, only a small amount of tumor growth may lead to cardiopulmonary deterioration. The 5 patients in this current series presented with large anterior-based neoplasms extending into the pleural space compressing the heart, great vessels, lung, or trachea. "Paradoxical" growth during chemotherapy resulted in cardiopulmonary deterioration and precluded safe administration and recovery from 4 planned cisplatin-based chemotherapy cycles. The large size of these neoplasms was underscored by the surgical approach, clamshell or combined sternotomy/thoracotomy, required for removal.

In 1997, Afifi and coworkers ${ }^{10}$ reported a patient with PMNSGCT who met the original GTS criteria. Two months after the patient received 4 cycles of cisplatin-based chemotherapy, cardiorespiratory deterioration secondary to tumor growth developed, and the patient underwent urgent surgery. Pathologic analysis of the mediastinal mass revealed mature teratoma only. The patient was alive and well after 2 years of follow-up. Other case reports of GTS in patients with PMNSGCT have found predominately mature teratoma with a mixed postchemotherapy pathology, challenging the concept that pathologic analysis of the growing mass needs to demonstrate mature teratoma only. ${ }^{11-14}$ Of note, only one of these more recent reports described a patient with PMNSGCT who required urgent surgery for cardiopulmonary deterioration before completion of planned chemotherapy. ${ }^{14}$ Long-term follow-up information was not available in this report. Pathology of the growing mass included in this present GTS series was mature teratoma in only 1 patient, with the other 4 patients demonstrating predominately teratoma with focal elements of immature teratoma, nonseminomatous germ cell, or nongerm cell cancer.

The combination of BEP has historically been the standard chemotherapy for "poor risk" nonseminomatous germ cell tumors, including PMNSGCT. A randomized trial comparing BEP with etoposide, ifosfamide, and cisplatin for "poor risk" nonseminomatous germ cell tumors, including PMNSGCT, demonstrated statistically equivalent survival. ${ }^{15}$ To eliminate the possibility of bleomycin-induced pulmonary toxicity before a major thoracic surgical procedure, we have used etoposide, ifosfamide, and cisplatin combination chemotherapy for patients with PMNSGCT over the past 10 years. Since initiating this change, there has been a significant reduction in postoperative respiratory failure after major thoracic surgery at the Melvin and Bren Simon Cancer Center. The potential for pulmonary complications 
is demonstrated by the single death in our series secondary to respiratory failure in an otherwise young healthy man who required urgent pneumonectomy immediately after receiving 3 courses of BEP chemotherapy.

\section{CONCLUSIONS}

GTS needs to be redefined for PMNSGCT on the basis of a current treatment strategy. GTS should include a small subset of patients with a growing mediastinal mass resulting in symptomatic compression of the great vessels/heart/lungs and decreasing serum tumor markers before or immediately after the fourth cycle of cisplatin-based chemotherapy, precluding safe completion and recovery from chemotherapy before surgery. In our series, symptomatic compression ranged from shortness of breath with any activity to frank cardiovascular collapse requiring intubation. Moreover, although mature teratoma is the predominate pathology of this subset, pathology is frequently mixed with foci of immature teratoma, germ cell, or non-germ cell cancer. Practitioners should have awareness for early recognition of this syndrome. Discontinuation of chemotherapy and referral for surgical therapy to prevent further cardiopulmonary deterioration are important. The surgical management of GTS is challenging. Despite the massive size of these neoplasms, resection with tumor-free margins can be accomplished. Completion of planned chemotherapy after recovery is recommended. Long-term survival is possible; however, careful surveillance is needed because early detection and resection of recurrent disease can still be curative.

\section{References}

1. Logothetis CJ, Sammuels ML, Trindade A, et al. The growing teratoma syndrome. Cancer. 1982;50:1629-35.

2. International Germ Cell Consensus Classification: a prognostic factor-based staging system for metastatic germ cell cancers. International Germ Cell Cancer Collaborative Group. J Clin Oncol. 1997;15:594-603.

3. Bokemeyer C, Nichols C, Droz J, et al. Extragonadal germ cell tumors of the mediastinum and retroperitoneum: results from an international analysis. $\mathrm{J} \mathrm{Clin} \mathrm{On}$ col. 2002;20:1864-73.

4. Hartmann JT, Nichols CR, Droz J, et al. Prognostic variables for response and outcome in patients with extragondal germ-cell tumors. Ann Oncol. 2002;13. 1017-28.

5. Schneider B, Kesler K, Brooks J, et al. Outcome of patients with residual germ cell or non-germ cell malignancy after resection of primary mediastinal nonseminomatous germ cell cancer. J Clin Oncol. 2004;2:1195-200.

6. Sarkaria IS, Bains MS, Sood S, et al. Resection of primary mediastinal nonseminomatous germ cell tumors: a 28-year experience at Memorial Sloan-Kettering Cancer Center. $J$ Thorac Oncol. 2011;6:1236-41.

7. Kesler KA, Einhorn LH. Multimodality treatment of germ cell tumors of the mediastinum. Thorac Surg Clin. 2009;19:63-9.

8. Kesler KA, Rieger KM, Hammoud ZT, et al. A twenty-five year single institution experience with surgery for primary mediastinal nonseminomatous germ cell tumors. Ann Thorac Surg. 2008;85:371-8.

9. Kesler KA. Technique of mediastinal germ cell tumor resection. Op Tech Thorac Cardiovasc Surg. 2009;14:55-65.

10. Afifi HY, Bosl GJ, Burt ME. Mediastinal growing teratoma syndrome. Ann Thorac Surg. 1997;64:359-62.

11. Chen LT, Chen CL, Hwang WS. The growing teratoma syndrome. A case of primary mediastinal nonseminomatous germ cell tumor treated with chemotherapy and radiotherapy. Chest. 1990;98:231-3.

12. Iyoda A, Hiroshima K, Yusa T, et al. The primary mediastinal growing teratoma syndrome. Anticancer Res. 2000;20:3723-6.

13. D'Aiuto M, Veronesi G, Peccatori FA, et al. Mediastinal-like growing teratoma syndrome. J Thorac Cardiovasc Surg. 2005;130:228-9.

14. Vohra LS, Talwar R, Mathur M, et al. Giant mediastinal teratoma-bull in china shop: management strategies. J Thorac Cardiovasc Surg. 2007;133:1382-3.

15. Hinton S, Catalano PJ, Einhorn LH, et al. Cisplatin, etoposide and bleomycin or ifosfamide in the treatment of disseminated germ cell tumors: final analysis of an intergroup trial. Cancer. 2003;97:1869-75. 